\section{Effects of different degrees of cataract on the multifocal electroretinogram}

\author{
W-K Tam¹, H Chan'1, B Brown² and M Yap ${ }^{1}$
}

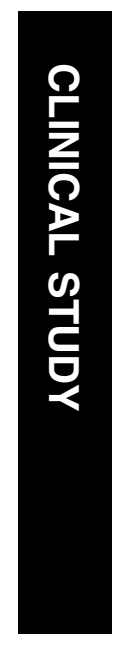

\begin{abstract}
Aim To study the effect of different degrees of nuclear cataract on the multifocal electroretinogram (mfERG).

Methods mfERGs were recorded from 30 elderly subjects with very mild, mild, or moderate nuclear cataracts using a VERIS System (version 4.1). The subjects were divided into three groups (10 in each group) according to their degree of nuclear cataracts as classified according to the Lens Opacities Classification System III (LOCS III). No subjects had any significant eye disease or degenerative changes except for cataracts. The mfERG responses were grouped into six concentric rings for analysis. Both the N1 and P1 amplitudes and the latencies of N1 and P1 of first-order responses were used for analysis. Results Amplitudes of N1 and P1 from the central retina $\left(14^{\circ}\right)$ were significantly reduced in patients with mild or moderate cataract when compared with subjects with very mild cataract. However, there was no significant reduction of $\mathrm{N} 1$ and $\mathrm{P} 1$ amplitudes in the paracentral retina $\left(14-40^{\circ}\right)$. There was no difference in the latencies of N1 and P1 in these three groups of subjects.

Conclusions The mfERG responses from the central retina (central $14^{\circ}$ ) were affected by the severity of cataract, but responses from the paracentral retina (14-40 $)$ were not affected. This suggests that in interpreting the mfERG in subjects with mild or moderate cataract subjects some care should be taken as reduced amplitudes (N1 and P1) will be expected from the central retina.
\end{abstract}

Eye (2004) 18, 691-696. doi:10.1038/sj.eye.6701318

Published online 13 February 2004

Keywords: multifocal electroretinogram; mfERG; cataract; LOCS III; aging;

light scattering
Introduction

The multifocal electroretinogram (mfERG) technique $^{1}$ allows simultaneous recording of many local retinal responses within a short time. It has been shown that numerous retinal eye diseases can be detected by the mfERG (eg diabetic retinopathy, glaucoma, retinitis pigmentosa). ${ }^{2-8}$ In these studies, it is reported that subjects had clear media. However, many patients with retinal eye disease (eg age-related macular degeneration) are elderly and some degree of lenticular change such as nuclear sclerosis is inevitable. For diagnostic purposes, it is important to know how changes in the ocular media would affect the mfERG topography.

It has previously been reported that media opacities such as cataract can reduce the amplitudes of the a-wave and b-wave of the scotopic flash ERG. ${ }^{9,10}$ Contradictorily, however, a larger than normal scotopic flash ERG response has also been recorded in patients with cataract. ${ }^{11}$ It was suggested that this might be due to the light-scattering effect (Ganzfeld effect) of the cataract. ${ }^{11}$ A recent study showed that a subject with mild cortical cataract had lower than normal mfERG responses. ${ }^{12}$

By using acrylic sheets or liquid-crystaldiffusers, the light-scattering effects of cataract have been simulated in mfERG studies. ${ }^{13,14}$ In a study on two subjects, Arai et al ${ }^{13}$ showed that central mfERG responses decreased slightly with increased scattering level, but that the peripheral responses did not show a corresponding reduction. Our own study on a larger group of young subjects showed that the central mfERG responses decreased significantly with increasing light scattering, but paradoxically the peripheral responses increased with increasing light scattering. ${ }^{14}$

In this study, we wanted to find out how cataract affects the mfERG.
${ }^{1}$ Department of Optometry and Radiography, The Hong Kong Polytechnic University Hong Kong

${ }^{2}$ School of Optometry, Queensland University of Technology

Queensland, Australia

Correspondence: $\mathrm{H}$ Chan Department of Optometry and Radiography, The Hong Kong Polytechnic University, Hong Kong

Tel: +85227667937

Fax: + 85227646051

E-mail: orhenry@

polyu.edu.hk

Presented in part at XXXXI ISCEV Conference, Nagoya Japan

Received: 6 May 2003 Accepted in revised form: 18 August 2003

Published online: 13 February 2004 


\section{Materials and methods}

\section{Subjects}

A total of 30 elderly subjects aged 50-75 years (mean age: 64 years) were recruited from the Optometry Clinic at The Hong Kong Polytechnic University. All subjects had mild to moderate nuclear cataract that was were classified and graded according to the Lens Opacities Classification System III (LOCS III) ${ }^{15}$ and their visual acuity (VA). A previous study has shown that there is a positive correlation between VA and LOCS nuclear cataract grade $(y=-0.23+0.0093 x)(y=\log$ MAR VA and $x=$ LOCS nuclear cataract grade). ${ }^{16}$

Subjects were divided into three groups with 10 subjects in each group according to the degree of cataract (see Table 1). All subjects had refractive errors of less than $\pm 3.00 \mathrm{D}$ and less than $1.00 \mathrm{D}$ of astigmatism. The mfERG topography in subjects with very mild nuclear cataract act as the normative values in this study, as subjects aged over 50 years old must have very mild nuclear cataract, which causes light scattering, ${ }^{17,18}$ even if it is not clinically significant and it does not affect VA.

To ensure that all subjects were free of retinal disease in the tested eye, all received an eye examination that included measurements of visual acuity and intraocular pressure. Ocular health was assessed by a slit-lamp examination and indirect ophthalmoscopy. Research procedures used in this study followed the tenets of the Declaration of Helsinki. All procedures were approved by the ethics committee of The Hong Kong Polytechnic University. Informed consent was obtained from all participating subjects after they were given an explanation of the study.

\section{Stimulus conditions}

The VERIS Science 4.1. system (Electro Diagnostic Imaging Inc., San Mateo, CA, USA) was used to record the mfERG. The stimulus matrix consisted of 103 scaled hexagonal elements presented on a Sony high resolution RGB 19" monitor (Sony, GPM-500P3, Japan) which had a frame rate of $75 \mathrm{~Hz}$. The stimulus was controlled by a video card (from EDI) in a Macintosh G3 computer. The stimulus hexagons were individually modulated between white $\left(165 \mathrm{~cd} / \mathrm{m}^{2}\right)$ and black $\left(3 \mathrm{~cd} / \mathrm{m}^{2}\right)$

Table 1 Subject group characteristics

\begin{tabular}{lccc}
\hline Group & $\begin{array}{l}\text { Best visual } \\
\text { acuity }\end{array}$ & $\begin{array}{l}\text { LOCS III } \\
\text { grading }\end{array}$ & $\begin{array}{l}\text { Mean age } \\
\text { (years) }\end{array}$ \\
\hline A & $6 / 6$ & 2 & 61 \\
B & $6 / 9$ & 4 & 65 \\
C & $6 / 12$ & 5 & 66 \\
\hline
\end{tabular}

according to a pseudorandom m-sequence. ${ }^{1}$ The luminance of the surround was set at $84 \mathrm{~cd} / \mathrm{m}^{2}$. A $0.8^{\circ}$ red fixation cross was used. This CRT monitor subtended a viewing angle of $41^{\circ}$ vertical and $53^{\circ}$ horizontal at a viewing distance of $40 \mathrm{~cm}$. The diameter of the different stimulus rings were: Ring $1-$ about $2.3^{\circ}$, Ring $2-$ about $2.3-7.8^{\circ}$, Ring $3-$ about $7.8-14^{\circ}$, Ring $4-$ about $14-22.4^{\circ}$, Ring 5 - about $22.4-31^{\circ}$, Ring $6-$ about $31-40^{\circ}$.

\section{Recording conditions}

The pupils were dilated with $1 \%$ tropicamide and all pupils were at least $6 \mathrm{~mm}$ before recording commenced. The Dawson-Trick-Litzkow (DTL) electrode was used as the active electrode. The reference and ground electrodes ( $\mathrm{Ag}-\mathrm{AgCl}$ electrode) were attached to the ipsilateral outer canthus and forehead respectively. The untested eye was occluded during recording. The testing distance was $40 \mathrm{~cm}$. Refractive errors were fully corrected for the viewing distance. The signals were amplified 100000 times with band-pass set at 3-300 Hz (Grass Instrument Co., Quincy, MA, USA). All mfERG responses were spatially smoothed once by averaging each local trace with $17 \%$ of each of its six nearest neighbours. A binary $\mathrm{m}$-sequence of $2^{15}$ was used for recording the mfERG. The total recording time was $7 \mathrm{~min} 17 \mathrm{~s}$, divided into 32 segments (recording periods). Subjects rested for a few seconds between segments. Any segment with breaks of fixation, eye movements, or blinks was discarded and recorded again. The recording conditions were performed according to ISCEV guidelines. ${ }^{19}$

\section{Analysis}

For data analysis, the mfERG responses were grouped in six concentric rings: responses with similar eccentricities from the central foveal response were grouped (Figure 1). In this study, the first-order kernel responses were analysed and only the amplitude and latencies of N1 and P1 were evaluated. We defined the first negative and

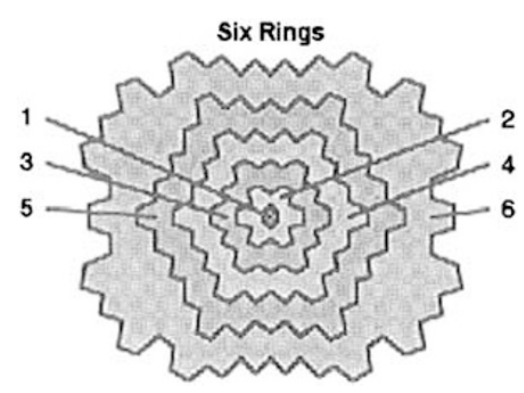

Figure 1 Responses were grouped into six rings for analysis. 
positive deflections of the mfERG as N1 and P1, respectively. The amplitude of $\mathrm{N} 1$ was measured from the baseline to the first negative peak. The amplitude of P1 was measured from the first negative peak to the first positive peak. The latencies of N1 and P1 were defined as the time period from the stimulus onset to the peak of N1 and P1 responses respectively. The effects of different degrees of cataract on mfERG responses were evaluated by using one-way ANOVA and Tukey HSD multiple comparisons. P-values less than 0.05 were considered statistically significant.

\section{Results}

The effects of different degrees of cataract on mfERG waveforms are shown in Figure 2. Table 2 shows the average mfERG waveform parameters and statistical findings (N1 latency, P1 latency, N1 amplitude, and P1 amplitude) when responses were grouped in six concentric rings. The $\mathrm{N} 1$ amplitudes from rings 1 to 3 were the lowest in moderate cataract group and highest for the very mild cataract group (Figure 3a). N1 amplitudes from ring 1 were significantly different among the three cataract groups. N1 amplitudes from rings 2 and 3 showed significant difference only between the very mild cataract and the moderate cataract groups.
There was no significant difference found from ring 4 to ring 6 for all three cataract groups.

The mean P1 amplitudes from rings 1 to 3 were the highest in the very mild cataract group and lowest for the moderate cataract group (Figure $3 b$ ). Table 2 shows that P1 amplitudes for rings 1 to 2 were also significantly different among the three cataract groups. For rings 3 and 4, P1 amplitudes were also significantly different between the very mild cataract group and the moderate cataract group. A significant difference of P1 amplitude between the mild cataract group and the moderate cataract group was also found in ring 4 . However, there was no significant difference among the three cataract groups in rings 5 and 6 . N1 and P1 latencies were not significantly different with increasing severity of cataract.

\section{Discussion}

A recent study on a single subject with cataract showed that mfERG responses were lower than in normal subjects of a similar age, but it was unclear whether the reduction was in the central retinal responses or peripheral retinal responses. ${ }^{12}$ Our study confirms and extends this finding: the reductions in mfERGs were related to the severity of nuclear cataract but only over central areas. In addition, our study found that the P2

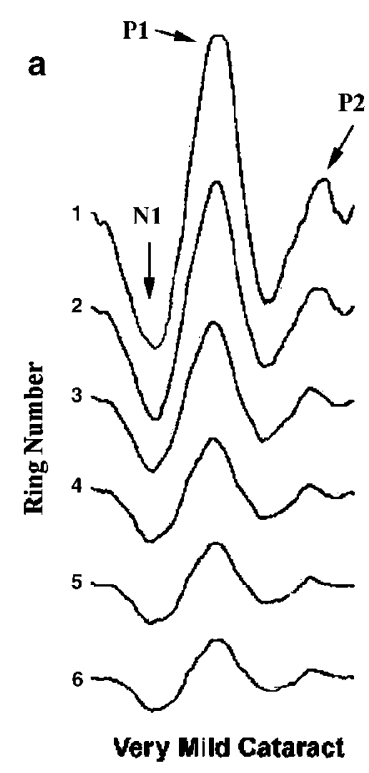

b

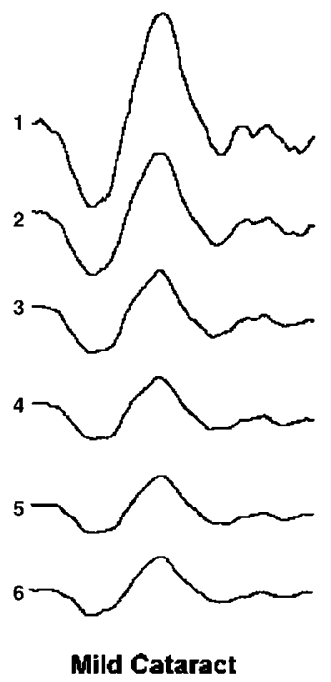

C

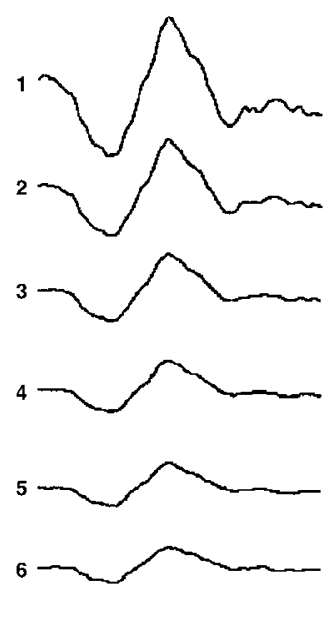

Moderate Cataract

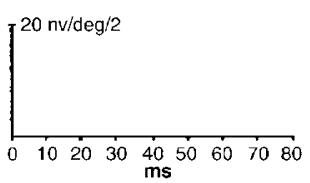

Figure 2 The mfERG from three of subjects for the six concentric rings: (a) Subject with very mild cataract, (b) Subject with mild cataract, (c) Subject with moderate cataract. N1 amplitudes from the central three rings (ie 1-3) were significantly lower with increasing degrees of cataract. P1 amplitude showed a similar trend. 
Table 2 Effect of different degrees of cataract on mfERG responses parameters and statistical findings: responses from six concentric rings

\begin{tabular}{|c|c|c|c|c|c|c|c|}
\hline & & Very mild & Mild cataract & Moderate & ANOI & $(d f=2)$ & \\
\hline $\mathrm{N} 1$ amplitude $\left(\mathrm{nV} / \mathrm{deg}^{2}\right)$ & Ring 1 & $28.90 \pm 6.52$ & $22.69 \pm 2.72$ & $13.63 \pm 4.23$ & $P=0.000^{*}$ & $F=26.075$ & $a, b, c$ \\
\hline & Ring 2 & $16.44 \pm 4.17$ & $12.69 \pm 3.42$ & $9.51 \pm 2.40$ & $P=0.000^{*}$ & $\mathrm{~F}=10.368$ & $\mathrm{~b}$ \\
\hline & Ring 3 & $9.64 \pm 2.29$ & $7.97 \pm 2.32$ & $6.45 \pm 1.57$ & $P=0.008^{*}$ & $F=5.843$ & $\mathrm{~b}$ \\
\hline & Ring 4 & $6.08 \pm 1.70$ & $6.07 \pm 1.52$ & $4.56 \pm 1.70$ & $P=0.076$ & $\mathrm{~F}=2.838$ & \\
\hline & Ring 5 & $4.78 \pm 1.51$ & $5.04 \pm 1.76$ & $3.66 \pm 1.38$ & $P=0.129$ & $\mathrm{~F}=2.214$ & \\
\hline & Ring 6 & $3.95 \pm 1.14$ & $3.94 \pm 1.55$ & $3.40 \pm 1.26$ & $P=0.557$ & $\mathrm{~F}=0.562$ & \\
\hline P1 amplitude (nV/deg $)$ & Ring 1 & $53.94 \pm 6.61$ & $44.55 \pm 4.07$ & $25.40 \pm 8.02$ & $P=0.000^{*}$ & $\mathrm{~F}=50.982$ & $a, b, c$ \\
\hline & Ring 2 & $28.10 \pm 5.15$ & $22.75 \pm 3.74$ & $17.46 \pm 4.90$ & $P=0.000^{*}$ & $\mathrm{~F}=13.178$ & $a, b, c$ \\
\hline & Ring 3 & $19.43 \pm 6.49$ & $15.81 \pm 4.38$ & $12.87 \pm 3.91$ & $P=0.025^{*}$ & $\mathrm{~F}=4.228$ & $\mathrm{~b}$ \\
\hline & Ring 4 & $12.47 \pm 2.28$ & $12.52 \pm 2.64$ & $9.39 \pm 3.07$ & $P=0.021^{*}$ & $\mathrm{~F}=4.466$ & $\mathrm{~b}, \mathrm{c}$ \\
\hline & Ring 5 & $9.42 \pm 2.56$ & $10.08 \pm 2.35$ & $8.42 \pm 1.89$ & $P=0.279$ & $\mathrm{~F}=1.340$ & \\
\hline & Ring 6 & $8.37 \pm 2.08$ & $8.04 \pm 3.01$ & $7.48 \pm 1.62$ & $P=0.688$ & $\mathrm{~F}=0.380$ & \\
\hline N1 latency (ms) & Ring 1 & $20.74 \pm 2.88$ & $19.42 \pm 2.09$ & $21.74 \pm 2.35$ & $P=0.126$ & $\mathrm{~F}=0.126$ & \\
\hline & Ring 2 & $19.58 \pm 1.88$ & $19.67 \pm 2.07$ & $21.25 \pm 2.47$ & $P=0.168$ & $F=0.168$ & \\
\hline & Ring 3 & $18.66 \pm 2.00$ & $20.14 \pm 2.74$ & $21.33 \pm 2.49$ & $P=0.065$ & $F=0.065$ & \\
\hline & Ring 4 & $18.91 \pm 1.71$ & $19.72 \pm 2.16$ & $20.75 \pm 1.98$ & $P=0.129$ & $\mathrm{~F}=0.129$ & \\
\hline & Ring 5 & $19.32 \pm 1.87$ & $20.74 \pm 1.62$ & $20.93 \pm 1.66$ & $P=0.091$ & $\mathrm{~F}=0.091$ & \\
\hline & Ring 6 & $19.17 \pm 2.14$ & $20.17 \pm 2.01$ & $21.59 \pm 2.38$ & $P=0.061$ & $\mathrm{~F}=0.061$ & \\
\hline P1 latency (ms) & Ring 1 & $37.73 \pm 2.51$ & $37.39 \pm 2.07$ & $38.56 \pm 1.92$ & $P=0.447$ & $\mathrm{~F}=0.761$ & \\
\hline & Ring 2 & $37.32 \pm 1.74$ & $36.49 \pm 2.03$ & $38.40 \pm 2.04$ & $P=0.107$ & $F=2.429$ & \\
\hline & Ring 3 & $37.32 \pm 0.66$ & $36.91 \pm 2.50$ & $38.73 \pm 1.96$ & $P=0.094$ & $\mathrm{~F}=2.584$ & \\
\hline & Ring 4 & $36.64 \pm 0.89$ & $36.98 \pm 1.41$ & $38.24 \pm 2.15$ & $P=0.074$ & $\mathrm{~F}=2.874$ & \\
\hline & Ring 5 & $37.64 \pm 1.89$ & $36.91 \pm 0.97$ & $38.40 \pm 1.93$ & $P=0.152$ & $\mathrm{~F}=2.023$ & \\
\hline & Ring 6 & $37.72 \pm 1.36$ & $36.65 \pm 1.29$ & $38.49 \pm 2.13$ & $P=0.058$ & $\mathrm{~F}=3.163$ & \\
\hline
\end{tabular}

Data are presented as mean \pm SD.

* Significant difference $(P<0.05)$ among three conditions

a Results differ significantly between very mild cataract and mild cataract $(P<0.05)$.

${ }^{\mathrm{b}}$ Results differ significantly between very mild and moderate cataract $(P<0.05)$.

'Results differ significantly between mild cataract and moderate cataract $(P<0.05)$.

amplitude with a latency of about $60 \mathrm{~ms}$ was greatly reduced in subjects with moderate cataract (Figure 2). Recent studies have pointed out that this P2 response is related to the first slice of second-order kernel response. ${ }^{720,21}$ In Figure 4, the first slice of second-order kernel response to demonstrate the effect of different degree of cataract can be seen. The first slice of secondorder kernel response was undetectable in subjects with moderate cataract. This could be the result of the reduction of the stimulus luminance and contrast.

It is well known that the amount of light scattering (both forward light scattering and backward light scattering) increases with increasing age. ${ }^{17,22,23}$ Light scattering occurs due to the presence of insoluble proteins in the lens. ${ }^{24}$ With increasing age, there is an increasing amount of insoluble proteins, so the amount of light scattering will increase as the criteria for Mie scattering are met. ${ }^{25}$ In the cataractous lens, forward light scattering reduces the contrast of retinal image.

Backward light scattering reduces the amount of light reaching the retina, as light is scattered back from the eye toward the light source. ${ }^{26}$
Previous studies have shown that mfERG responses (P1 amplitude) from the central and para-central retina decreased linearly when stimulus luminance is decreased. ${ }^{4,12,27,28}$ Brown and $\mathrm{Yap}^{27}$ showed that the mfERG responses decreased linearly at all retinal eccentricities when the stimulus contrast was decreased. On the basis of these reports, cataract should reduce central and peripheral mfERG responses. However, in our study, we found that central retinal responses were decreased but peripheral retinal responses were not. This finding is slightly different from the report by Chan et al. ${ }^{14}$ They found that central mfERG responses were reduced, but peripheral retinal responses were increased under light-scattering conditions. It is not clear why peripheral retinal responses were not affected by cataract in our study, but we can conclude generally that light-scattering caused by media opacities can affect the amplitude of mfERG responses. ${ }^{11,14}$

There have been several studies on the effect of aging on mfERG topography. ${ }^{28-32}$ Mohidin et al. ${ }^{29}$ found that the decrease in response density in aged eyes was mainly at the central $10^{\circ}$ diameter of the macular with no 
significant reduction in peripheral responses. However, the oldest subject in their study was only 52 years old. Nabeshima et al..$^{30}$ reported although the reduction of response density was greatest at the central retina, peripheral retinal responses also decreased with
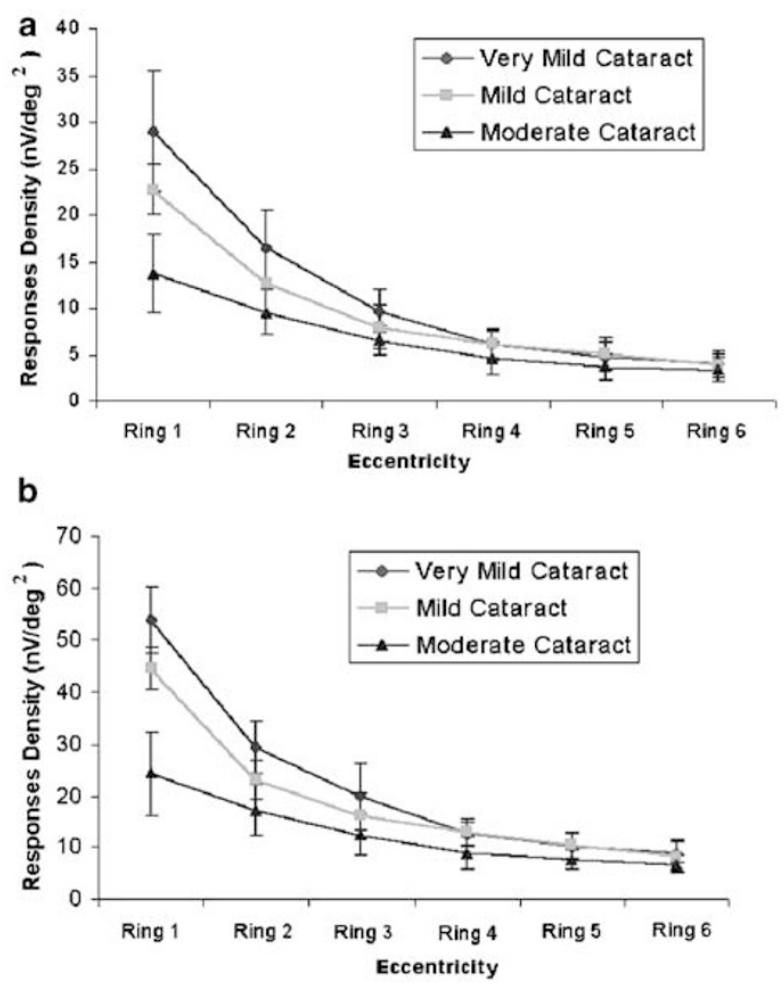

Figure 3 (a) Mean N1 amplitudes of the six concentric rings for three groups of subjects with cataract. (b) Mean P1 amplitudes of the six concentric rings for the three groups of subjects with cataract. Error bars are \pm 1 standard deviation. increasing age. Since the study from Nabeshima et al. included subjects with slight nuclear opacity, it is not clear whether the age-related effects on the mfERG were due to neural factors or optical factors. Fortune and Johnson $^{28}$ adjusted their mfERG data for the effect of reduced lens transmission and pupil diameter in aged subjects to rule out the effect of optical factors and they found that the decline of mfERG responses with age could be attributed to optical factors. They concluded that neural factors only played a small role that was mainly restricted to the central retinal responses $\left(5^{\circ}\right)$. Another study based on calculating the effect of media opacities (decrease stimulus luminance and contrast) on mfERG topography indicated that the effect of aging on mfERG is due to both optical and neural factors. ${ }^{32}$ However, two recent studies strongly claimed that smaller mfERG responses in elderly were due to neural factors rather than optical factors. ${ }^{31,33}$ The results of these studies, therefore, suggest that each laboratory should establish normative values for older adults. $^{28-33}$

The results of our study suggest that presence of cataract should be taken into consideration in the clinical application of mfERG. These findings further suggest that each laboratory should also establish its normative values for different degrees of cataract if the clinician wishes to use the mfERG to assess retinal function behind a cataract.

\section{Acknowledgements}

This study was supported by Competitive Earmark Research Grant (PolyU 5277/01 M) from The Research Grants Committee of the Hong Kong SAR.

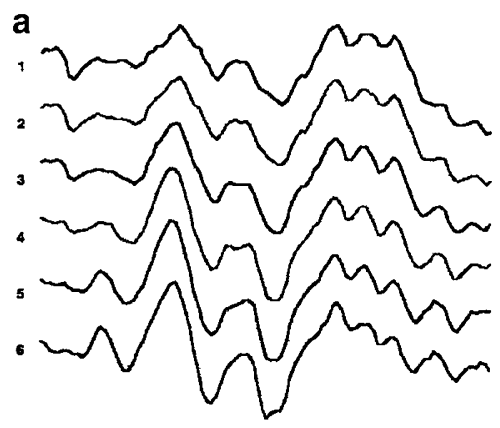

Very Mild Cataract

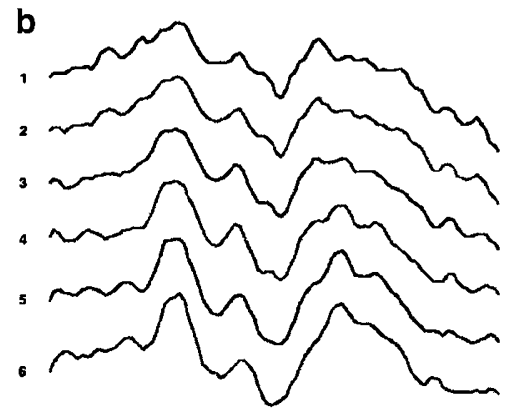

Mild Cataract

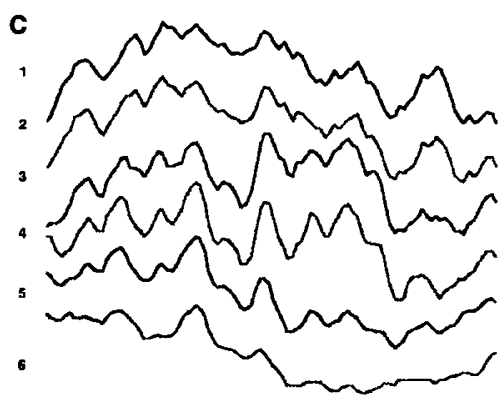

Moderate Cataract

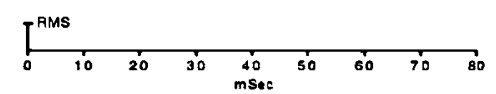

Figure 4 First slice of the second-order responses waveforms for the six concentric rings from three subjects: (a) Subject with very mild cataract, (b) Subject with mild cataract, (c) Subject with moderate cataract. The first slice of second-order responses is not obvious in subject with moderate cataract. 


\section{References}

1 Sutter EE, Tran D. The field topography of ERG components in man-I. The photopic luminance response. Vision Res 1992; 32: 433-446.

2 Fortune B, Schneck ME, Adams AJ. Multifocal electroretinogram delays reveal local retinal dysfunction in early diabetic retinopathy. Invest Ophthalmol Vis Sci 1999; 40: 2638-2651.

3 Chan HH, Brown B. Pilot study of the multifocal electroretinogram in ocular hypertension. Br J Ophthalmol 2000; 84: 1147-1153.

4 Chan HL, Brown B. Investigation of retinitis pigmentosa using the multifocal electroretinogram. Ophthalmic Physiol Opt 1998; 18: 335-350.

5 Chan HL, Brown B. Multifocal ERG changes in glaucoma. Ophthalmic Physiol Opt 1999; 19: 306-316.

6 Palmowski AM, Allgayer R, Heinemann-Vemaleken B. The multifocal ERG in open angle glaucoma - a comparison of high and low contrast recordings in high-and low-tension open angle glaucoma. Doc Ophthalmol 2000; 101: 35-49.

7 Hood DC. Assessing retinal function with the multifocal technique. Prog Retin Eye Res 2000; 19: 607-646.

8 Kretschmann U, Seeliger MW, Ruether K, Usui T, Apfelstedt-Sylla E, Zrenner E. Multifocal electroretinography in patients with Stargardt's macular dystrophy. Br J Ophthalmol 1998; 82: 267-275.

9 Hurst MA, Douthwaite WA. Assessing vision behind cataract - a review of methods. Optom Vis Sci 1993; 70: 903-913.

10 Fishman GA. The electroretinogram. In: Fishman GA, Sokol S, Brich DG, Holder GE, Brigell MG, (eds). Electrophysiology in Disorders of the Retina, Optic Nerve, and Visual Pathway. The Foundation of the American Academy of Ophthalmology, San Francisco; CA, 2001, pp 1-155.

11 Galloway NR. Electrophysiological testing of eyes with opaque media. Eye 1988; 2(Part 6): 615-624.

12 Yoshii M, Yanashima K, Wakaguri T, Sakemi F, Kikuchi Y, Suzuki $\mathrm{S}$ et al. A basic investigation of multifocal electroretinogram: reproducibility and effect of luminance. Jpn J Ophthalmol 2000; 44: 122-127.

13 Arai M, Lopes de Faria JM, Hirose T. Effects of stimulus blocking, light scattering, and distortion on multifocal electroretinogram. Jpn J Ophthalmol 1999; 43: 481-489.

14 Chan HL, Siu AW, Yap MK, Brown B. The effect of light scattering on multifocal electroretinography. Ophthalmic Physiol Opt 2002; 22: 482-490.

15 Chylack Jr LT, Wolfe JK, Singer DM, Leske MC, Bullimore MA, Bailey IL et al. The Lens Opacities Classification System III. The Longitudinal Study of Cataract Study Group. Arch Ophthalmol 1993; 111: 831-836.
16 Elliott DB, Situ P. Visual acuity versus letter contrast sensitivity in early cataract. Vision Res 1998; 38: 2047-2052.

17 Siik S, Airaksinen PJ, Tuulonen A. Light scatter in aging and cataractous human lens. Acta Ophthalmol (Copenh) 1992; 70: 383-388.

18 Smith GT, Smith RC, Brown NA, Bron AJ, Harris ML. Changes in light scatter and width measurements from the human lens cortex with age. Eye 1992; 6(Part 1): 55-59.

19 Marmor MF, Hood DC, Keating D, Kondo M, Seeliger MW, Miyake Y. Guidelines for basic multifocal electroretinography (mfERG). Doc Ophthalmol 2003; 106: 105-115.

20 Sutter E. The interpretation of multifocal binary kernels. Doc Ophthalmol 2000; 100: 49-75.

21 Shimada Y, Horiguchi M. Stray light-induced multifocal electroretinograms. Invest Ophthalmol Vis Sci 2003; 44: 1245-1251.

22 Hemenger RP. Light scatter in cataractous lenses. Ophthalmic Physiol Opt 1990; 10: 394-396.

23 Whitaker D, Steen R, Elliott DB. Light scatter in the normal young, elderly, and cataractous eye demonstrates little wavelength dependency. Optom Vis Sci 1993; 70: 963-968.

24 Kamei A, Iwata S, Horwitz J. Characterization of water-insoluble proteins in human lens nuclei. Jpn J Ophthalmol 1987; 31: 433-439.

25 Heavens OS, Ditchburn RW Insight into Optics. John Wiley \& Sons, New York, 1991.

26 Elliott DB. Evaluating visual function in cataract. Optom Vis Sci 1993; 70: 896-902.

27 Brown B, Yap MK. Contrast and luminance as parameters defining the output of the VERIS topographical ERG Ophthalmic Physiol Opt 1996; 16: 42-48.

28 Fortune B, Johnson CA. Decline of photopic multifocal electroretinogram responses with age is due primarily to preretinal optical factors. J Opt Soc Am A: Opt Image Sci Vis 2002; 19: 173-184.

29 Mohidin N, Yap MK, Jacobs RJ. Influence of age on the multifocal electroretinography. Ophthalmic Physiol Opt 1999; 19: $481-488$

30 Nabeshima T, Tazawa Y, Mita M, Sano M. Effects of aging on the first and second-order kernels of multifocal electroretinogram. Jpn J Ophthalmol 2002; 46: 261-269.

31 Jackson GR, Ortega J, Girkin C, Rosenstiel CE, Owsley C. Aging-related changes in the multifocal electroretinogram. J Opt Soc Am A Opt Image Sci Vis 2002; 19: 185-189.

32 Gerth C, Garcia SM, Ma L, Keltner JL, Werner JS. Multifocal electroretinogram: age-related changes for different luminance levels. Graefes Arch Clin Exp Ophthalmol 2002; 240: 202-208.

33 Seiple W, Vajaranant TS, Szlyk JP, Clemens C, Holopigian K, Paliga J et al. Multifocal electroretinography as a function of age: the importance of normative values for older adults. Invest Ophthalmol Vis Sci 2003; 44: 1783-1792. 